\title{
Performance Evaluation of Patient Monitoring System With Different Routing Protocols
}

\author{
Ravi Tiwari \\ Dept. of Electrical Engg. \\ National Institute of Technology \\ Rourkela, India
}

\author{
Sonam Shrivastava \\ Dept. of Electrical Engg. \\ National Institute of Technology \\ Rourkela, India
}

\author{
Susmita Das \\ Dept. of Electrical Engg. \\ National Institute of Technology \\ Rourkela, India
}

\begin{abstract}
In order to improve the control overhead and to achieve the reliable data transmission in the patient monitoring system based on ZigBee, here the concentration is given on the comparative performance evaluation of different routing algorithm in a wireless body area network (WBAN) using the OPNET simulation tools. WBAN contains the number of wireless mobile nodes forming an unpredictable topology and link instability that make routing a core issue. This paper provides a relative analysis of AODV, DSR, and OLSR routing protocols with increasing number of wireless nodes in WBAN on the basis of end to end delay, load, and throughput. Simulation results demonstrate that OLSR routing protocol is performing best amongst all protocols under an increasing number of node scenario.
\end{abstract}

\section{General Terms}

WBAN, Patient Monitoring, AODV, DSR, OLSR, OPNET.

\section{Keywords}

Throughput, end to end delay, load, control overhead.

\section{INTRODUCTION}

Over the past few years, there is a rapid growth in the wireless body area network (WBAN) for patient monitoring system in information processing and wireless data transmission. WBAN provides low cost wireless sensor network technology that creates a system to monitor patient, remotely using an Internet or intranet, and it could be seen as a special-purpose wireless sensor node network that provide the health monitoring to anyone, anytime and anywhere [1].

Figure 1 shows the overall architecture of a WBAN system model for remote patient monitoring environment. In WBAN, networks of wireless sensors are placed very close to the body for collection of specific physiological data from it. Those sensors forward data to ZigBee router and then it transfers to a ZigBee coordinator who allows the medical specialist to continuously monitor the patient situation by comparison with an original database of the patient [3]. Inpatient monitoring system, data transmission is very important and that is why IEEE formed a specialist study group IEEE 802.15.6, working on the development of body area network $[14,15]$.

The main function of such devices is to collect patient physiological data and forward to medical center in the efficient way. Therefore, routing is a very important task in WBAN. Nowadays, lots of routing algorithms are available, but it is difficult to select the suitable algorithm for desired network.

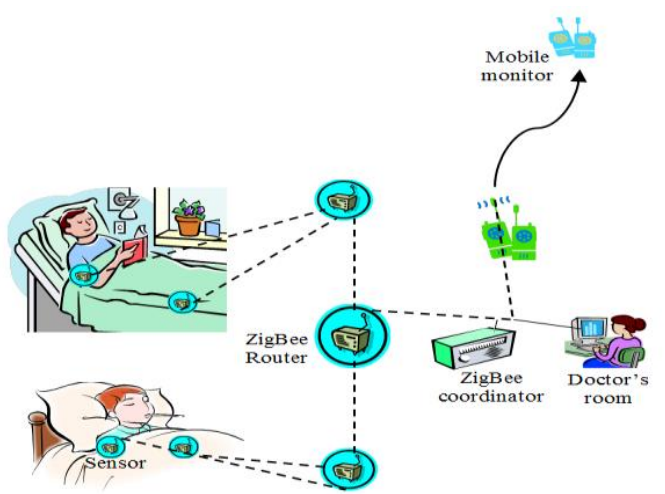

Figure 1. Architecture of patient monitoring system

Many challenges, including a medical data transmission error and how to provide better healthcare services in underserved areas, are taken into account [2]. Here, performance evaluation of body area network has been done on the basis of various routing approaches to reduce the informational error on the basis of end to end delay, load, and throughput for reliable delivery of packets of information $[3,4,6]$.

This paper is organized as follows. In section 2, the classification of routing protocol for patient monitoring system has been described. The complete process of DSR, AODV, and OLSR routing protocols explained in section 3. Simulation of routing algorithms on the basis of end to end delay, load, and throughput are analyzed in section 4 . The conclusions are drawn in section 5 .

\section{CLASSIFICATION OF ROUTING PROTOCOLS FOR PATIENT MONITORING SYSTEM}

A routing protocol specifies how the routers communicate with each other. Figure 2 depicts the broad classification of routing protocols with examples. 


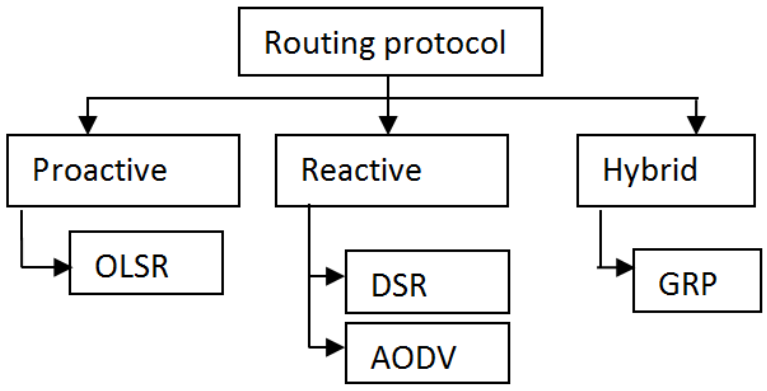

Figure 2. Routing protocol classification

\subsection{Proactive Routing Protocol}

This type of routing protocol continuously modifies its routing table by sending request update to neighbor node and a sharing routing table, so the route is already available when any node wants to send a packet of information to a destination node. Here, latency delay is low as the route is established before communication requirement.

\subsection{Reactive Routing Protocol}

In this type of Routing protocol when any node wants to send a packet of information to a destination node, it will first check whether the route is available in the routing table or not, if the route is available, the source node sends through that route otherwise it sends a request for a route from source node to destination.

\subsection{Hybrid Routing Protocol}

This protocol combines reactive and proactive routing protocols along with a location assisted routing protocol.

\section{COMPARISON OF PROCESS FOR DIFFERENT ROUTING PROTOCOL}

\subsection{DSR (Dynamic Source Routing)}

DSR is a reactive and on demand routing protocol, which supports unicast routing. It is a source routing protocol means that the source knows the complete hop sequence to the destination, and it can be used in multi hop wireless ad- hoc network. DSR self-organizing and configuring the network by two processes:

\section{1) Route discovery process.}

2) Route maintenance process.

The router has maintained the table of recently the seen requests for avoiding the memory overhead and repetition of the same route requests

\subsubsection{Route discovery process}

When a node wants to send a packet of information to the destination, then the source node, firstly search the route to the destination in its route cache. If it finds a route, it uses that route to send its packet of information otherwise it initiates the Route Request Message (RREQ) having a unique identification number and address of the source and destination by flooding method [7]. The intermediate nodes in this flooding method receive the RREQ message and resend to its neighbor by adding their own address. As the destination node receives the RREQ, it will initiate a Route Reply (RREP) packet with full route information to the sender. The route considers by the destination for RREP is the shortest path taken by RREQ packet. Now a source has complete route information, and it can start routing of packets.

\subsubsection{Route maintenance process}

A destination node sends an acknowledgement (ACK) to the sender when the message received successfully, otherwise it will send Route Error Message (RERR) which indicates the problem in transmission.

As DSR is reactive and on demand routing protocol, a major problem is its non-uniform packet size so intermediate node may not be able to forward the packets of information correctly [6].

\subsection{AODV (Ad hoc On demand Distance Vector)}

The AODV routing protocol is on demand and destination based routing protocol. AODV is also a reactive routing protocol as DSR but the main difference is that in AODV, RREQ carries the destination address, whereas in DSR it carries full routing information; this implies that AODV has potentially less routing overhead. Another difference is that in AODV, RREP carries the destination IP address and sequence number, whereas in DSR, it carries the address of every node along the route, so AODV resolves the problem of potential overhead found in DSR [7].

When a node wants to send a packet of information to destination node subsequently it broadcasts a RREQ on the network. When the intermediate node receives the RREQ message, it creates a reverse route back to the destination node and then checks for a valid route to the destination in the route table, if it does not have a valid route, it will broadcast the RREQ message in the network.

Lifetime is related to the entry in route table if a route is not used for a long time, then that route is deleted from route table. When the destination node receives the RREQ, it generates Route Reply (RREP). If the number of route reply is received at the source, then the route with the shortest hop count is chosen. In case when the intermediate node fails to forward the packet to next hop or destination due to any reason, it generates the Route Error (RERR) message. When the source node receives the RERR, it will again start the route discovery process for destination [7].

The advantage in AODV routing is that it provides an easy way to get change in link situation, but the node may experience the large delays during route construction and consume more bandwidth as the network size increases [5].

\subsection{OLSR (Optimized Link State Routing protocol)}

OLSR is a proactive routing protocol for Ad-hoc network, hence has the advantage of having the routes immediately available when needed. The main feature of the OLSR routing protocol is to decrease control overhead by reducing the number of broadcasts as compared to the flooding method by selecting some nodes for broadcasting. Those selected nodes are called as Multi-Point Relays (MPR) [9]. As only MPR node will take part in retransmission of packets of information during the flooding mechanism, it will significantly reduce the number of re-transmission [10].

OLSR selects MPR, such that it will provide optimal routes in terms of the number of hops. It is a table driven protocol and continuously exchanges the information about topology with 
all nodes of the network. This protocol is generally best for large and dense network.

\section{SIMULATION ANALYSIS OF ROUTING PROTOCOL}

In this paper, network simulator OPNET (Optimized Network Engineering Tool) is used for system performance evaluation. In this paper, network simulator OPNET (Optimized Network Engineering Tool) is used for system performance evaluation. The development language used is $\mathrm{C}$ [8]. The simulation is performed with the random waypoint mobility issue at HTTP traffic.

Simulation has been performed by considering the random waypoint mobility model. In this type of model, the position of nodes is randomly selected within a specified area and after that, nodes move to selected position in the linear form with random speed.

The technology used is a WLAN with operation mode $802.11 \mathrm{~b}$ having $11 \mathrm{Mbps}$ data rate and transmission power $5 \mathrm{~mW}[12]$. We have run this scenario in the context of $100 \mathrm{~m} \times 100 \mathrm{~m}$ with 30 nodes and 100 node networks for 300 seconds; each node has a speed of $5 \mathrm{~m} / \mathrm{s}$ [13]. The network topology model shown in the Fig. 3, 4 and OPNET MANET station node model is shown Fig. 5.

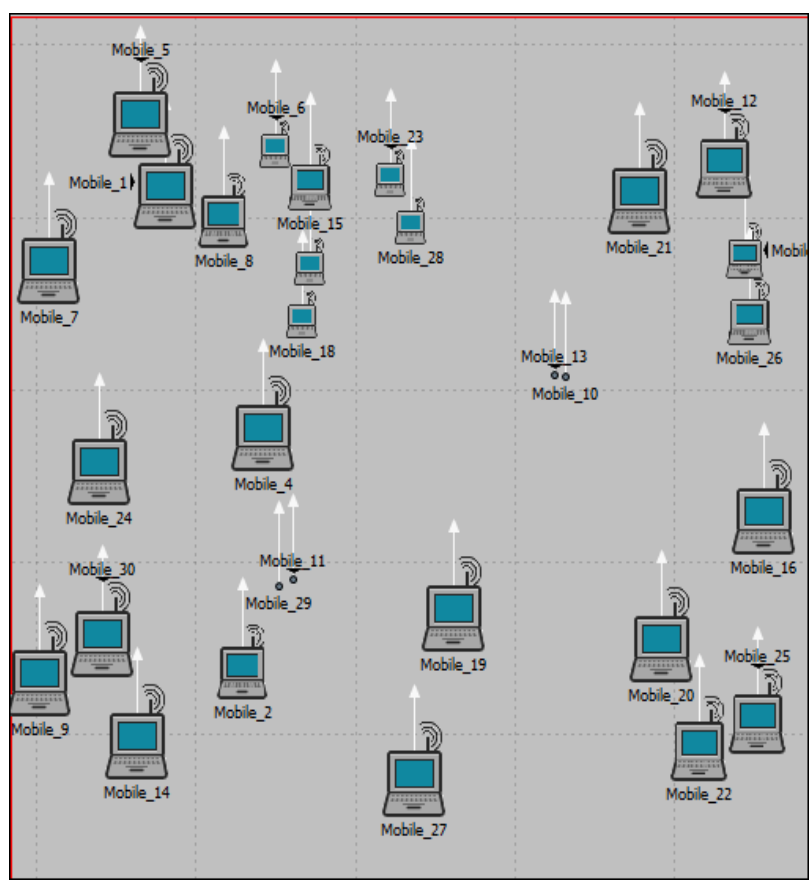

Figure 3. Network model with 30 nodes

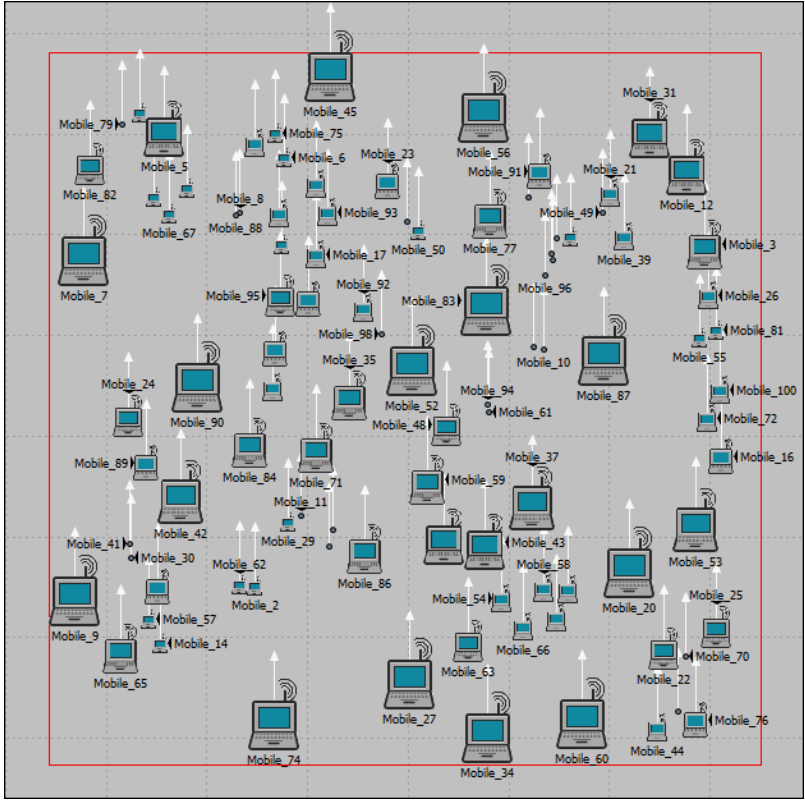

Figure 4. Network model with 100 nodes

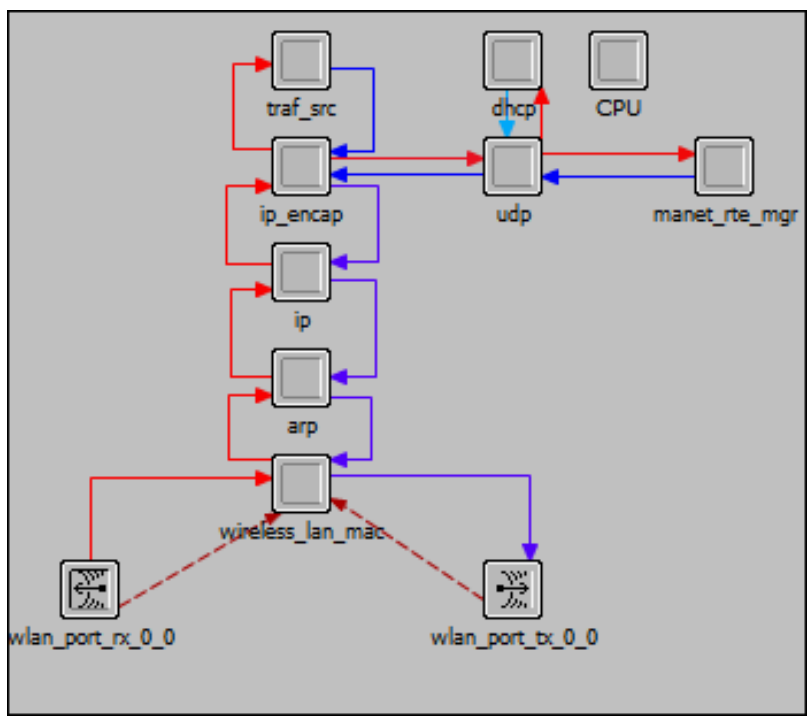

Figure 5. Manet station node model

The performance parameter for evaluating routing protocol for patient monitoring systems are:

1. End to end delay $\left(\mathbf{T}_{d}\right)$ :It represents time taken by all the packets to travel from source to the destination. It is given as:

$\mathrm{T}_{\mathrm{d}}=$ Processing Delay $\left(\mathrm{P}_{\mathrm{d}}\right)+$ QueuingDelay $\left(\mathrm{Q}_{\mathrm{d}}\right)+$ Transmission delay $\left(\mathrm{T}_{\mathrm{d}}\right)$

Where,

Processing Delay =Time takes to process packet header.

Transmission Delay $=$ Time it takes to push the packet onto the link.

Propagation Delay $=$ Time for signal to reach its destination. 
The End to end delay comparison curve with respect to simulation time for 30 and 100 node system model are shown in Figure 6 and Figure 7 respectively.

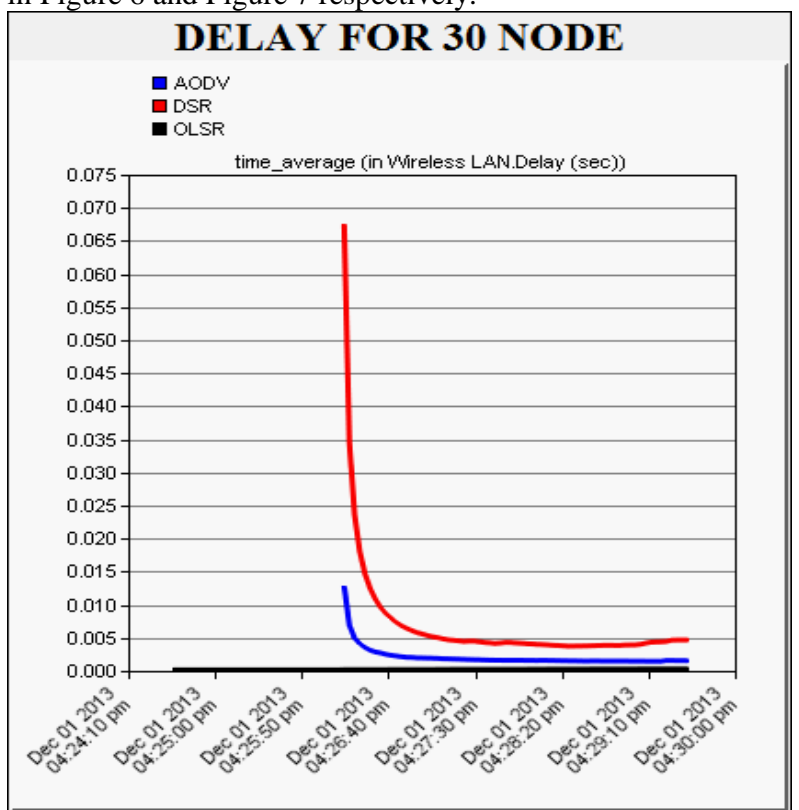

Figure 6. End to end delay $(\mathrm{Sec})$ in different routing for 30 node network.

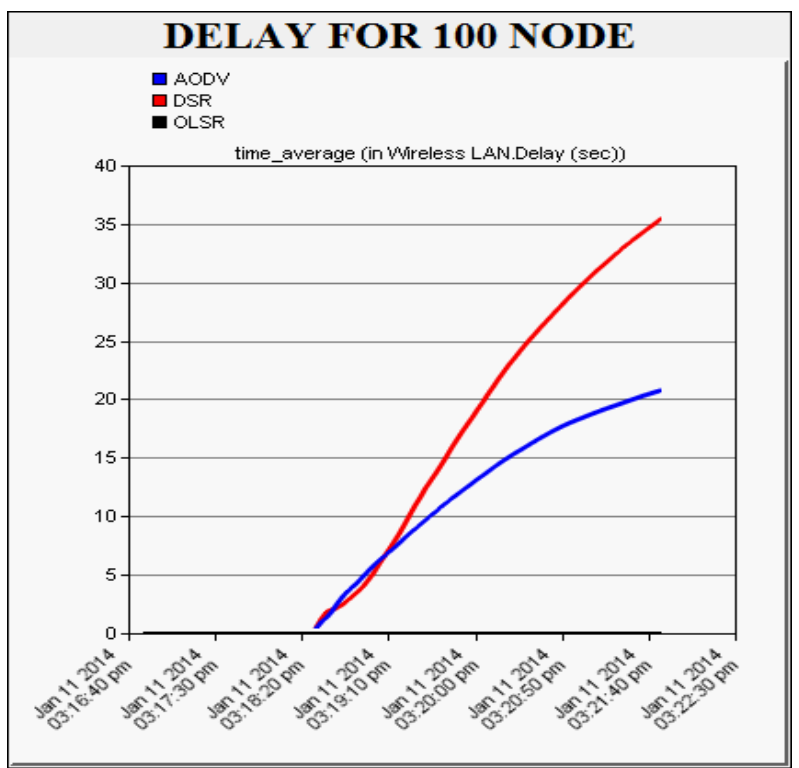

Figure 7. End to end delay (Sec) in different routing for 100 node network

Figure 6 and Figure 7 shows that in the beginning of the simulation routing cache has not been established, and gradually it establishes a routing table through the route discovery process.

2. Load :It represents the total load in bits/Sec submitted to WLAN layer by all higher layers in all WLAN nodes of the network. If the load is more, it will create more traffic in the network, which is difficult to handle. If the load in the network is high, it will slow down the speed of packet and hence increasing the collision of control packets resulting in slow stabilization of routing a packet.
The LOAD comparison curve with respect to simulation time for 30 and 100 node system models are shown in Figure 8 and Figure 9 respectively.

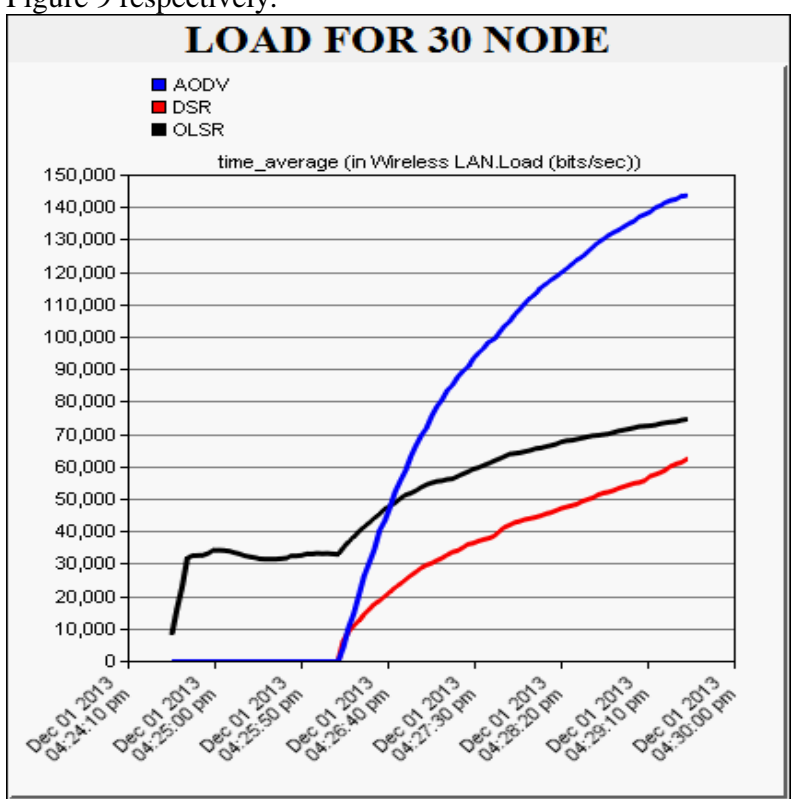

Figure 8. LOAD (bits/Sec) in different routing protocol for 30 node network

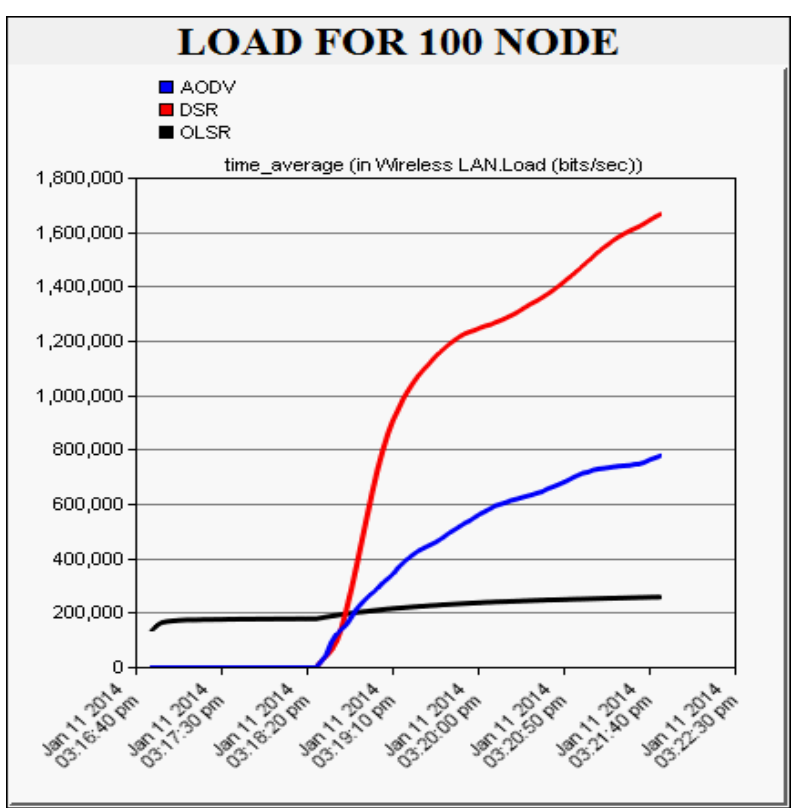

Figure 9. . LOAD (bits/Sec) in different routing protocol for 100 node network

3. Throughput: It represents the average rate of successful packet of information received at the destination. It is also defined as the entire data received by destination node from source divided by the total time takes by destination to get the last packet.

Throughput $=\frac{\text { Number of delivered packet } \times \text { packet size } \times 8}{\text { Total duration of simulation }}$ 
The Throughput comparison curve with respect to simulation time for 30 and 100 node system model are shown in Figure 10 and 11 respectively.

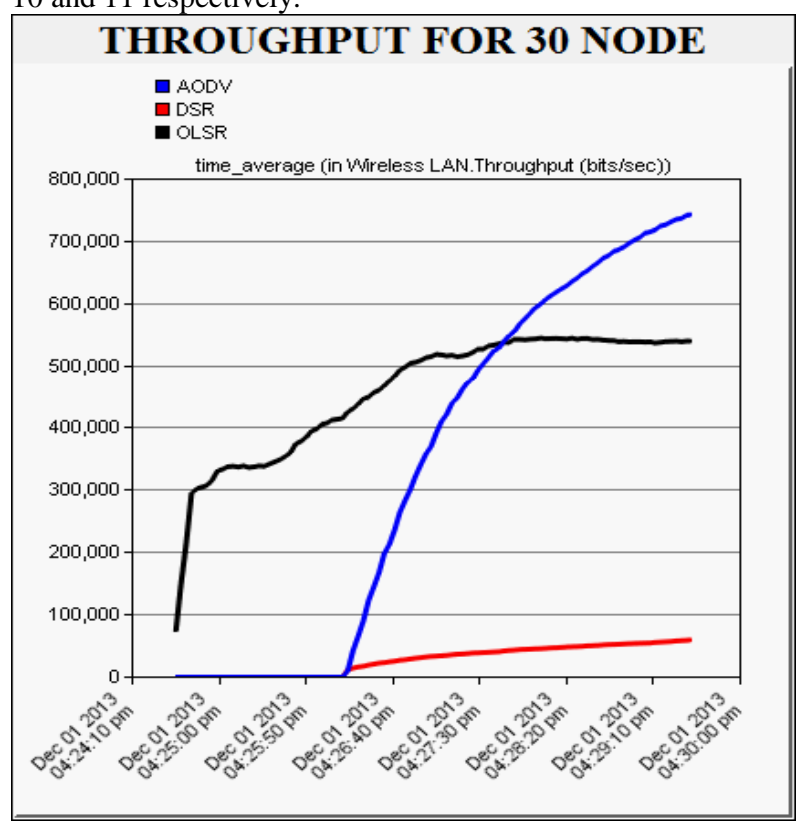

Figure 10. Throughput (bits/Sec) in different routing protocol for 30 node network

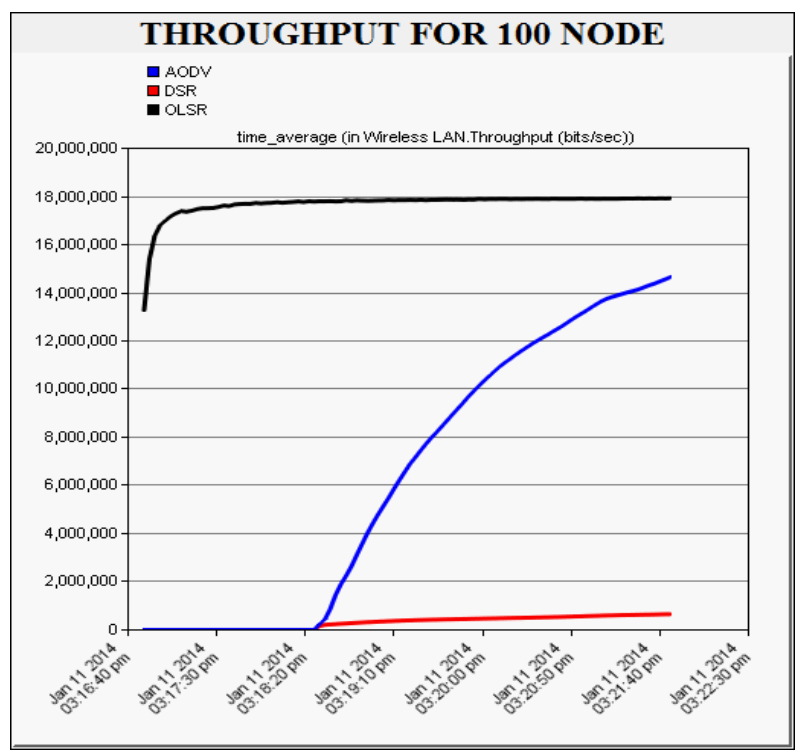

Figure 11. Throughput (bits/Sec) in different routing protocol for 100 node network

Table1. Simulation results comparison (in terms of rank) For 30 nodes System model

\begin{tabular}{|c|c|c|c|}
\hline Protocol & Delay & Load & Throughput \\
\hline AODV & $2^{\text {nd }}$ & $3^{\text {rd }}$ & $1^{\text {st }}(\max )$ \\
\hline DSR & $3^{\text {rd }}$ & $1^{\text {st }}(\min )$ & $3^{\text {rd }}$ \\
\hline OLSR & $1^{\text {st }}(\min )$ & $2^{\text {nd }}$ & $2^{\text {nd }}$ \\
\hline
\end{tabular}

From the Table 1, simulation result shows that for 30 nodes, WBAN system model end to end delay are the minimum in case of OLSR routing protocol whereas throughput is maximum for AODV routing protocol.

Table2. Simulation results comparison (in terms of rank) For 100 nodes System model

\begin{tabular}{|c|c|c|c|}
\hline Protocol & Delay & Load & Throughput \\
\hline AODV & $2^{\text {nd }}$ & $2^{\text {nd }}$ & $2^{\text {nd }}$ \\
\hline DSR & $3^{\text {rd }}$ & $3^{\text {rd }}$ & $3^{\text {rd }}$ \\
\hline OLSR & $1^{\text {st }}(\min )$ & $1^{\text {st }}(\min )$ & $1^{\text {st }}(\max )$ \\
\hline
\end{tabular}

From the Table 2, simulation result for 100 nodes WBAN system model shows that, the OLSR routing protocol is best as end to end delay is minimum and throughput is the maximum among other routing protocols.

\section{CONCLUSION}

In this paper, the comparative study of three routing protocols AODV, DSR, and OLSR has been done on the basis of end to end delay, load, and throughput. The two scenarios first with 30 nodes and other with 100 nodes has been simulated for the WBAN patient monitoring system model. The results are found in the graphical form by using OPNET simulator 14.5. Here, it can be concluded that OLSR routing protocol performs the best among all the routing protocols with increasing number of nodes in the WBAN system model because in this protocol, routes are immediately available when needed, and it also significantly lessened the control overhead by reducing the number of re-transmission.

\section{ACKNOWLEDGMENTS}

The authors would like to thank their friends and colleagues for supporting and encouraging them during their work. Finally, authors reserve the most special gratitude for their families because without their patience this would be imposible.

\section{REFERENCES}

[1] Jamil Y. Khan, Mehmet R. Yuce and Farbood Karami, "Performance evaluation of wireless body area sensor network for remote patient monitoring" $30^{\text {th }}$ annual international IEEE EMBS conference vancouver, British Columbia, Canada, August 2008.

[2] Lingwei Zhang, Hanjun Jiang and Jainjun Wei, "A reconfigurable sliding IF tranceiver for $400 \mathrm{MHz} / 2.4$ $\mathrm{GHz}$ IEEE 802.15.6/ ZigBee WBAN hubs with only $21 \%$ tuning range VCO" IEEE journal of solid state circuits, vol. 48, NO. 11, November 2013.

[3] Muhannad Quwaider and Suber Biswas, "On body packet routing algorithms for body sensor networks" first international conference on network and communication, 2009.

[4] Nikolaos A. Pantazis, Stefanos A. Nikolidakis and Dimitrios D Vergados, "Energy efficient routing protocols in wireless sensor networks: A survey" IEEE communications surveys and tutorials, vol. 15, No.2, second quarter, 2013. 
[5] Zahian Ismail and Rosilah Hassan,"Effect of packet size on AODV routing protocol implementation in homogeneous MANET" Third international conference on computational intelligence, modelling and simulation, 2011.

[6] Asma Tuteja, Rajneesh Gujral, and Sunil Thalia, "Comparative performance analysis of DSDV, AODV, and DSR routing protocol in MANET using NS2" International conference on advance in computer engineering, 2010.

[7] Qutaiba Razoui, Ahmed Boushehri, Mohamed Goballah, and Lina Alsaleh, "Extensive simulation performance analysis for DSDV, DSR and AODV MANET routing protocols" $27^{\text {th }}$ international conference on advanced information networking and application workshops, 2013.

[8] Hammoodi, I. S., et al. "A comprehensive performance study of OPNET modeler for ZigBee wireless sensor networks" Next Generation Mobile Applications, Services and Technologies, 2009. NGMAST'09. Third International Conference on. IEEE, 2009.

[9] Mohamed ELshaish, Nidal Kamal and Azlan Awang, "High throughput routing algorithm metric for OLSR routing protocol in wireless mesh networks" International colloquium on signal processing and its applications (CSPA), 2009.
[10] Tinku Rasheed, Usman Javid, Mosz Jerbi and Khaldoun AL Agha, "Scalable Multi-hop Ad Hoc routing using modified OLSR routing protocol" The $18^{\text {th }}$ annual IEEE international symposium on personal, Indoor and mobile radio communication, 2007.

[11] Joseph Dorleus, Ralph Holweck, Zin Ren, Hongbin Li, Hong-Liang Cui, and Joun Mendina, "Modeling and simulation of fading and pathloss in OPNET for range communication" 1-4244-0445-2/07/, 2007.

[12] Kai Yang nad Jainfing Ma, "Implementation of IEEE 802.1x in OPNET" IEEE 978-1-4244-1787-1787-2/08, 2008.

[13] M.S. Hasan, H. Yu, A. Carrington, T.C. Yang, "Cosimulation of wireless networked control system over mobile ad hoc network using simulink and OPNET" published in IET communication DOI: 10.1049/ietcom.2008.0536.

[14] Ting, Kok Seng, et al. "The performance evaluation of IEEE 802.11 against IEEE 802.15. 4 with low transmission power" Communications (APCC), 17th Asia-Pacific Conference on. IEEE, 2011.

[15] N. F. Timmons and W. G. Scanlon, "Improving the ultra low power performance of IEE 802.15.6 by adaptive synchronisation" publisher in IET wireless sensor systems doi: 10.1049/iet-wss-2011.0036. 